\title{
Recurrent Tetanus in Diagnosed Breast Cancer Patients of the Philippine General Hospital
}

\author{
Jesi Ellen C. Bautista, Leah L. Shiong Shu, Jose Leonard R. Pascual and Paul Matthew D. Pasco
}

Department of Neurosciences, College of Medicine and Philippine General Hospital, University of the Philippines Manila

\begin{abstract}
This is the first report of recurrent tetanus in two cases of patients with intraductal carcinoma of the breast. Both patients were treated with anti-tetanus vaccines and appropriate antibiotics. There was no surgical intervention. The patients were discharged well after resolution of the tetanus. However, both patients had recurrence of the tetanus several weeks from discharge. We explored the possible causes and prevention of tetanus recurrence in immunocompromised patients with chronic wounds.
\end{abstract}

Key Words: recurrent tetanus, tetanus, breast cancer, cancer

\section{Introduction}

Tetanus is a real and pressing public health concern in the Philippines. From 2001 to 2008 alone, the Philippine National Statistics Office quoted a total of 4,220 cases of tetanus reported in government centers, or a yearly average of 527.5 cases. ${ }^{1}$ This number is an underestimate of the actual number of cases in the country since it only includes figures from government centers and does not include neonatal tetanus. There is no data on the incidence of tetanus in breast cancer patients. Tetanus is associated with altered cardiovascular, respiratory, and renal physiology. Physical and psychological sequelae have been reported and mortality may be as high as $10-50 \%$ in developing countries. ${ }^{2,3}$

We report here two cases of recurrent tetanus in patients with breast cancer whose presumed infectious foci were the breast mass wounds. In this subset of the population afflicted with chronic wounds and in states of immunosuppression, the recommended management of tetanus proved to be inadequate in preventing recurrence. Given the high mortality of tetanus, it is useful to investigate what additional measures may be needed to avoid

\footnotetext{
Corresponding author: Jesi Ellen C. Bautista, MD

Department of Neurosciences

Philippine General Hospital

University of the Philippines Manila

Taft Avenue, Ermita, Manila 1000 Philippines

Telefax No.: +632 5548462

Email: jesi.bautista@gmail.com
}

recurrence. In this report we explore the possible causes for tetanus recurrence as well as the management of tetanus specifically in immunocompromised patients with chronic wounds. This report has been registered with the Expanded Hospital Research Office of UP-PGH.

\section{Case Presentation}

\section{Case 1}

A 40-year-old female diagnosed with intraductal breast carcinoma stage III-C presented at the emergency department with trismus. She had undergone neoadjuvant chemotherapy, radical mastectomy, and adjuvant 35-day course of radiotherapy. However, the tumor recurred 1 month prior and developed ulceration. At the emergency department, she presented with dysphagia and spontaneous spasms of the extremities. She was admitted as a case of tetanus. She was immediately given 10,000 units equine tetanus antitoxin and $0.5 \mathrm{~mL}$ tetanus toxoid vaccine. Prophylactic tracheostomy was performed. A 14-day course of intravenous metronidazole was given at $500 \mathrm{mg}$ every 6 hours. Intravenous diazepam and oral baclofen were started for spasm control. Initial laboratory data revealed her WBC count to be at 10,900/L. Wound aspirate grew Pseudomonas aeruginosa and Staphylococcus epidermidis. Debridement of the wound on the breast mass was deferred due to anticipated excessive bleeding as well as possible tumor seeding. The patient was discharged 24 days later with no signs and symptoms of trismus, spasticity, spasms, dyspnea, or dysphagia. She was advised to have the second and third tetanus toxoid vaccines a month apart.

Forty days after discharge from the hospital, the patient again experienced trismus, dysphagia, neck rigidity and fever. She was admitted at the emergency department for recurrent tetanus. Spontaneous spasms and respiratory distress were not observed. The mass on her right breast still had ulcerations with foul-smelling discharge. She had not received the recommended booster shots of tetanus vaccine. Ten thousand units of tetanus antitoxin and $0.5 \mathrm{~mL}$ tetanus antibodies were immediately administered. Metronidazole, diazepam, and baclofen were initiated. Laboratory tests revealed: WBC 28,400/L and creatine kinase total of $<20$ (N: 21-232 U/L). The patient underwent another tracheostomy. The breast mass was cleaned regularly but no debridement 
was done for fear of tumor seeding. The patient was transferred to the intensive care unit but expired in 24 hours due to dysautonomia.

\section{Case 2}

A 54-year-old female was brought to the emergency room for 1-week history of difficulty swallowing and limb spasms. She was managed as a case of tetanus. She had progressively enlarging bilateral breasts masses the past 9 months. The left breast mass developed ulcerations 2 months prior. On admission the patient manifested with trismus, dysphagia, and neck rigidity with no spontaneous spasms and no dyspnea. She was given 10,000 units of equine antitetanus immunoglobulin and $0.5 \mathrm{~mL}$ tetanus toxoid vaccines. A 14-day course of intravenous metronidazole at $500 \mathrm{mg}$ every 6 hours was initiated together with piperacillintazobactam at $4.5 \mathrm{~g}$ every 8 hours. The spasms were managed with diazepam, baclofen, and magnesium sulfate infusions. She underwent tracheostomy. Initial laboratory tests revealed WBC of $12,500 / \mathrm{L}$, creatinine of $128 \mathrm{umol} / \mathrm{L}$, and negative urine myoglobin. Wound culture did not grow any organism and blood cultures grew Staphylococcus hominis. The patient refused any intervention on her breast masses. She was discharged 25 days later asymptomatic. There was no mention of subsequent anti-tetanus vaccines administered in the interim.

Thirty-two days after discharge, the patient noted increased swelling and erythema of the left breast mass associated with occasional spasms of the abdominal and trapezius muscles and was readmitted as a case of recurrent tetanus. She did not have any trismus, dysphagia or dyspnea. Three thousand units of tetanus immunoglobulin was administered. Intravenous metronidazole was started for tetanus and intravenous ceftriaxone was started for concurrent pneumonia. To control the spasms, diazepam, baclofen, and magnesium sulfate infusion were initiated early on, with addition of chlorpromazine and levetiracetam later. Initial laboratory tests during this admission revealed WBC of 10,600/L and creatinine of 6 . Blood cultures were negative. Wound cultures grew Providencia stuartii and Klebsiella pneumoniae. Histopathology result of an incision biopsy revealed intraductal carcinoma of the breast. Mastectomy was offered but not pursued during this admission. The patient was discharged 43 days later asymptomatic.

\section{Discussion}

The diagnosis of tetanus is entirely clinical with no characteristic laboratory findings. The World Health Organization recommends at least one of the following signs for diagnosis: trismus, risus sardonicus, or painful muscular contractions. ${ }^{4}$ Common points of entry include bites, burns, puncture wounds, intravenous drug use, and non-healing wounds. In $15-25 \%$, no recent wounds can be identified ${ }^{4}$.
There are some reports of tetanus in patients with tumors including soft tissue sarcoma, uterine leiomyoma, and ulcerating giant phyllodes breast tumor., ${ }^{5,6}$

To our knowledge, this is the first published report of recurrent tetanus in patients with diagnosed breast cancer. In both cases, the likely sites of infection were chronic ulcerated breast masses. The chronicity of the wound was a risk factor that made these individuals prone to primary and repeat tetanus infections.

A report has estimated the number of tetanus cases in chronic wounds to be $11-14 \% .^{7}$ In diabetic patients, for example, diabetic foot ulcers account for $25 \%$ of contracted tetanus. ${ }^{8}$ Severe tetanus has also developed from biopsy of chronic masses. ${ }^{9}$ Recurrent tetanus has also been reported in chronic IV drug users. ${ }^{10}$

It is possible that what is called recurrence may actually be a relapse of the tetanus due to persistence or reactivation of infection in the presumed focus. ${ }^{11}$ However, many of the observed cases of recurrence have wounds that have already healed. Other possible mechanisms for recurrence are reinfection of the original wound and infection at a second site. $^{12}$

In the two cases in our report, though the presumed foci were still present, symptoms of tetanus manifested again after they had already been symptom-free for several weeks. They have thus been deemed recurrences. Nevertheless, as only $30 \%$ of documented cases have cultures positive for $C$. tetani, true recurrence could not be proved based on wound cultures alone. ${ }^{4}$ In both our cases, C. tetani did not grow in the wound cultures.

There have been several reports of recurrent tetanus in developed and developing countries alike. In the Philippines, tetanus cases are reported without distinguishing whether they are primary, relapse, or recurrent. The arbitrary period of 1 month has been proposed to distinguish recurrence from relapse but there has been no evidence to support this. ${ }^{13}$ The period reported for recurrences varies from 1 week to several years. At the moment, data is lacking on the incidence, prognostic factors, and appropriate treatment for recurrent tetanus.

One example of recurrent tetanus reported in the 1950s was in a 38-year-old female who contracted tetanus following a tooth extraction and again from a cut six and a half years later. ${ }^{14}$ She was treated during her first admission with 160,000 units of passive tetanus antitoxin followed by injections of 1,500 units every 4 days and one dose of tetanus toxoid. She was discharged cured. On the second admission, she expired after ventricular fibrillation developed on the eighth hospital day. It is notable that a bout of tetanus infection and a single tetanus toxoid shot were not enough to confer lasting or effective immunity in an apparently healthy individual. In a separate report, attempts were made to objectively analyze toxin and antitoxin titers in three patients with tetanus recurring 3, 6, and 12 months after the 
first episode. ${ }^{12}$ The authors also concluded that there is little or no long-lasting effective immunity conferred by an attack of tetanus or by injection of passive vaccine. Several other reports show similar findings, all on presumed previously healthy subjects. ${ }^{15,16}$ In another case, a previously healthy 60year-old female was given 20,000 units passive vaccine and $10 \mathrm{Lt}$ (flocculation units) tetanus toxoid on admission and a booster after 30 days only to develop relapse on the 46th hospital day. Tetanus antibody titer taken on the 54th day was $0.0092 \mathrm{IU} / \mathrm{mL}$, significantly lower than the protective titer of $0.01-1 \mathrm{IU} / \mathrm{mL}$ despite the patient having been given two doses of tetanus toxoid vaccine. In this case, two doses of tetanus toxoid were not sufficient to mount effective immunity within 54 days of administration of the first dose in a patient with no signs of immunosuppression. ${ }^{17}$

A single dose of tetanus toxoid ensures little or no protection even among immunocompetent subjects. ${ }^{4}$ Immunity develops only 2-4 weeks after the second dose and even then about $10 \%$ of patients, labeled "bad responders", remain poorly protected. Moreover, immunity decreases over time, with the percentage of poorly protected persons increasing after just 1 year. It is the third dose that induces significant levels of antitoxin such that protection can last at least 5 years. ${ }^{4}$ Additional doses given at least 1 year after the last dose prolongs immunity even further, lasting 10 years after the 4 th dose and 20 years after the 5 th. This suggests that giving tetanus toxoids during bouts of tetanus and on follow up will only protect the individual from tetanus at the very earliest 2 weeks after the 2 nd dose is given. Hence, to prevent recurrent tetanus, the focus should be to prevent primary tetanus from occurring in the first place. Otherwise, an individual previously not vaccinated against tetanus is prone to recurrence within 6 weeks of being given the first tetanus toxoid vaccine.

There are no studies yet on the incidence of tetanus in those who have received adequate vaccination. The most recent recommendation of the US Centers for Disease Control and Prevention (CDC) and the World Health Organization (WHO) for primary tetanus prevention is to give three doses of tetanus vaccine 1 month apart with additional boosters every 10 years for all individuals over 10 years of age. ${ }^{4}$ Individuals diagnosed with tetanus are also given three doses of anti-tetanus vaccines. Guidelines on tetanus vaccination do not include confirmation of immunity by antibody titer determination.

For patients with cancer, the risk for tetanus may be increased not only by the presence of chronic wounds but also by their immunosuppressed states. One of the mechanisms tumors may employ to evade the immune system is to produce factors that modulate the functions of immune cells. There are several immunosuppressive factors that can be produced by tumors, targeting both innate and adaptive immune systems causing alterations in components such as T cell and NK cell. ${ }^{18}$ Also, the psychological stress alone of having cancer contributes to the alteration and depression of the immune system. ${ }^{19}$ This suppression of the immune system is similarly seen in those with diabetes, HIV, or who are on chronic immunosuppressants in whom antibody titers may not rise normally in response to vaccination. $^{20}$ In individuals with or prone to immunosuppression, vaccination is recommended before immunosuppression sets in or until improvement in immunity is seen. However, in tetanus treatment, the same schedule, dose, and indications for vaccination are applied whether the individual is immunocompromised or not.

The probable site of infection must be treated adequately to prevent relapse or recurrence. This can be a challenge in cancer patients presenting with difficult-to-treat chronic wounds. There are no recommendations for the prevention of tetanus in this population. A report on a case of chronic radiation-induced mandibular osteomyelitis provided support for chronic metronidazole administration to prevent tetanus recurrence. ${ }^{21}$ The patient was discharged clinically cured and was maintained on metronidazole 500 $\mathrm{mg}$ daily for 14 months. The patient received a total of three doses of tetanus toxoid vaccine. Eight months after metronidazole was discontinued, he had another bout of tetanus. This case is evidence of the inability of the recommended vaccine regimen to confer effective and longlasting immunity in cancer patients with chronic wounds. Cases of chronic wounds may warrant periodic administration of antitoxin until the wound is healed or until active immunity has been confirmed. ${ }^{12}$

\section{Conclusion}

Tetanus remains a prominent infectious disease in the Philippines. In this paper, we presented two individuals with chronic malignant breast masses who had repeated bouts of tetanus. The presence of chronic wounds that can be foci for repeated infection or states of immune compromise may be factors to developing recurrent tetanus infections. There is data on the inability of some individuals to mount sufficient immunity even after the recommended regimen of tetanus vaccines has been given and cancer patients may fall into this category. Further studies are needed to determine the optimum tetanus vaccination regimen and the frequency of anti-tetanus titer determination in cancer patients as well as explore the factors leading to recurrent tetanus infections. More data is also needed on the incidence of recurrent tetanus in adequately vaccinated individuals. One study has shown evidence for the efficacy of chronic metronidazole administration in preventing tetanus recurrence in patients with chronic wounds. This has the potential to decrease the morbidity in cancer patients but further evidence is needed to support its validity and inclusion in the guidelines for tetanus management. 


\section{Recurrent Tetanus in Breast Cancer Patients}

\section{References}

1. National Statistical Coordination Board, Notifiable Diseases Reported by Cause: 2001-2008 [Online]. 2012 [cited 2012 Nov]. Available from http://www.nscb.gov.ph/secstat/d_vital.asp

2. Illis LS, Taylor FM. Neurological and electroencephalographic sequelae of tetanus. Lancet. 1971; 1(7704):826-30.

3. Cook TM, Protheroe RT, Handel JM. Tetanus: a review of literature. Br J Anaesth. 2001; 87(3):477-87.

4. World Health Organization. The Immunological Basis for Immunization Series Module 3: Tetanus. 1993: pp 1-21.

5. Coulson AS, Lee H. Tetanus in a patient with a uterine tumor. Am Surg. 1976; 42(3):215-8.

6. Yamashita S, Karashima R, Tsushima A, Tawara S, Kawahara K. Tetanus caused by ulcerated giant phyllodes tumor. Breast J. 2007; 13(6):614-5.

7. Texier S, Dermu M. Tetanus in a man presenting with a chronic wound. Presse Med. 2004; 33:759-60.

8. Rogers LC, Frykberg RG. Tetanus prophylaxis for diabetic foot ulcers. Clin Podiatr Med Surg. 2006; 23:769-75.

9. Aranegui B, Florez A, Garcia-Doval I, Garcia-Cruz A, De La Torre C, Cruces M. Generalised Tetanus in a patient with a chronic ulcerated skin lesion. BMJ. 2010; 340:97-9.

10. Iqbal N. Tetanus in I.V. heroin users. Ann Saudi Med. 2001; 21(5-6):2969.

11. Alhady SM. Recurrent tetanus: report of a case. Med J Aust. 1961; 48(2):219-20.

12. Vakil BJ, Mehta AJ, Tulpule TH. Recurrent Tetanus. Postgrad Med J. 1964; 40:601-3.

13. Vener HI, Bower AG. Tetanus: Second attack with recovery. JAMA. 1940; 114:2198

14. Cain HD, Falco FG. Case Reports: Recurrent tetanus. Calif Med. 1962; 97(1):31-3.

15. Lindley-Jones M, Lewis D, Southgate JL. Recurrent tetanus. Lancet. 2004; 363:2048.

16. Wickramasinghe SY, Fernando M. Recurrent tetanus. Br Med J. 1967; 4:(5578)530.

17. Bhatt AD, Dastur FD. Relapsing tetanus (a case report). J Postgrad Med. 1981;27:184-6.

18. Whiteside TL. Immune suppression in cancer: Effects on immune cells, mechanisms and future therapeutic intervention. Semin Cancer Biol. 2006; 16:3-15.

19. Reiche EMV, Nunes SOV, Morimoto HK. Stress, depression, the immune system, and cancer. Lancet Oncol. 2004; 5(10):617-25.

20. Opravil M, Fierz W, Matter L, Blaser J, Luthy R. Poor antibody response after tetanus and pneumococcal vaccination in immunocompromised, HIV-infected patients. Clin Exp Immunol. 1991; 84:185-9.

21. Wakasaya $\mathrm{Y}$, Watanabe M, Tomiyama M, et al. An unusual case of chronic relapsing tetanus associated with mandibular osteomyelitis. Intern Med. 2009; 48(15):1311-3 